\title{
Can detection of Braf p.V600E mutation be improved? Comparison of allele specific multiplex sequencing to present tests
}

\author{
Thuraiayah Vinayagamoorthy*1, David Zhang ${ }^{2}$, Fei Ye ${ }^{2}$, Dilanthi Vinayagamoorthy ${ }^{1}$, Roger Hodkinson ${ }^{1}$ \\ ${ }^{1}$ MultiGEN Diagnostics Inc., Greensboro, United States \\ ${ }^{2}$ Department of Pathology, Mount Sinai School of Medicine, New York, United States
}

Received: March 8, 2017

DOI: $10.5430 /$ jst.v7n2p14
Accepted: April 5, 2017

URL: https://doi.org/10.5430/jst.v7n2p14
Online Published: April 12, 2017

\begin{abstract}
Objective: This is an investigative study to evaluate a new companion diagnostic platform, allele specific multiplex sequencing (ASMS). Detection of Braf p.V600E from solid tumors is used as the test model with the following objectives: 1) whether ASMS can detect Braf p.V600E/K mutations from a variety of solid tumors, 2) whether ASMS can detect all Braf p.V600E from samples that were positive for Braf V600E by SNaPshot or Ion Torrent, and 3) whether ASMS can detect Braf p.V600E among samples that were reported negative by SNaPshot or Ion Torrent.

Methods: ASMS is a novel modification (US Patent 6197510) of traditional Sanger sequencing, with Lower Limit of Detection (LLOD) of $20 \mathrm{GE}$ (Genome Equivalent) and $0.001 \%$ sensitivity. We compared ASMS to clinical samples previously tested either by SNaPshot or Ion Torrent methods.

Results: We analyzed 83 DNA extracts from FFPE samples (41 tested by SNaPshot and 42 tested by Ion Torrent). There was a total of thirty-seven samples positive for Braf p.V600E (16 by Ion Torrent; 21 by SNaPshot), and all of these samples tested positive by ASMS for Braf p.V600E. Out of the 46 negatives for Braf p.V600E (20 by SNaPshot; 26 by Ion Torrent samples), ASMS detected Braf p.V600E positive results in 10 of the SNaPshot and in 18 of the Ion Torrent negative samples. ASMS could detect both Braf p.V600E and the wild-type Braf p.V600 simultaneously with 40 pg of FFPE DNA extracts.

Conclusions: ASMS assay detected all Braf p.V600E positives from different types of solid tumors that previously tested positive by SNaPshot or Ion Torrent. Further, ASMS was able to detect Braf p.V600E among samples that were reported negative by SNaPshot or Ion Torrent.
\end{abstract}

Key Words: Ultra-sensitive, Companion diagnostics, Allele specific multiplex sequencing (ASMS), Braf p.V600E, Vemurafenib

\section{INTRODUCTION}

Chemotherapy is an essential component of cancer treatment, to eliminate residual tumor burden following surgery and radiation therapy. Discoveries of cancer specific somatic mutations have led to the development of targeted chemotherapies, which selectively act on cancer cells..$^{[1,2]}$ Hence, predicting the potential effectiveness of chemotherapy (chemo-prediction) has become a standard procedure in the selection of chemotherapeutics. ${ }^{[3]}$ Presently, chemoprediction is based on identification of specific somatic point

*Correspondence: Thuraiayah Vinayagamoorthy; Email: Moorthy@multigen-diagnostics.com; Address: MultiGEN Diagnostics Inc., 1100 Revolution Mill Drive, Greensboro, NC 27405, United States. 
mutations (SPMs) in tumors that are known to respond to a specific chemotherapeutic drug. In response to the question: Could treatment outcomes with Braf p.V600E kinase inhibitors be improved? Our answer is potentially 'Yes', provided Braf p.V600E mutations could be more efficiently detected.

Detection of Braf p.V600E mutation is a qualitative test and is routinely tested in stage IV melanoma on FFPE (Formalin-Fixed Paraffin-Embedded) samples. Patients with Braf p.V600E positive results are selected for treatment with kinase inhibitors (e.g. Vemurafenib). Detection of Braf p.V600E in FFPE samples face two challenges: 1) Detection that is inclusive of low abundance Braf p.V600E mutations, and 2) detection of Braf p.V600E mutations in a heterogeneous cell population with a significantly greater number of normal cells (e.g. FFPE samples). Tumor mass is a result of a single or few cancer cells proliferating over a certain period. Further, during metastasis, the breakaway cancer cell(s) lodge at secondary sites and proliferate to become a tumor mass. In both scenarios, the rate of proliferation, the size of tumor mass and the number of copies of Braf p.V600E is unpredictable. Given these variables, there is a need to use a highly sensitive assay that can detect low abundance Braf p.V600E mutations in order to potentially enhance treatment outcomes, as additional patients will be identified as potentially qualifying for treatment with kinase inhibitors. ${ }^{[4,5]}$ In attempting to meet these objectives, methods with significantly increased sensitivities and specificities have been developed, including allele-specific PCR. ${ }^{[6-13]}$ Although allele-specific PCR and ASMS both use allelespecific primers, they are two quite different methods. In allele-specific PCR, allele-specific primers are used in the amplification step. This is followed by identification of the generated amplicon using a labeled nucleotide probe. In contrast, with ASMS, allele-specific primers are used in the identification step (sequencing step) after amplification, generating a Braf p.V600E specific nucleotide sequence that can be verified. ${ }^{[14]}$ Preliminary analytical validation of ASMS shows a lower limit of detection (LLOD) of $20 \mathrm{GE}$ for Braf p.V600E, and a sensitivity ratio of mutant to wild-type of 1: 100,000 .

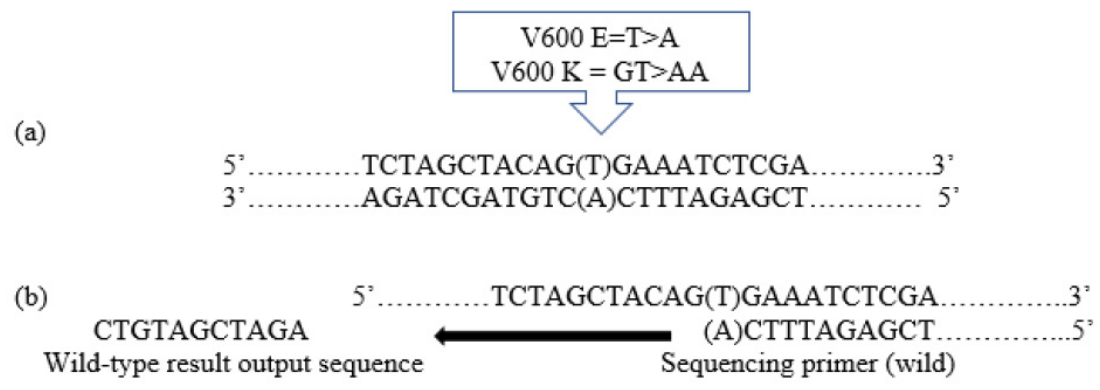

(c)

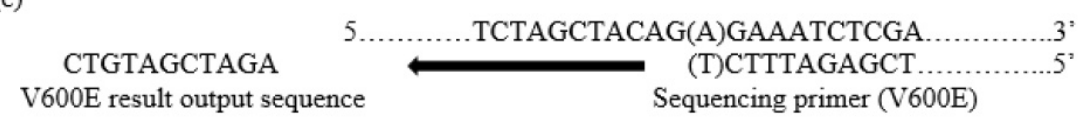

(d)

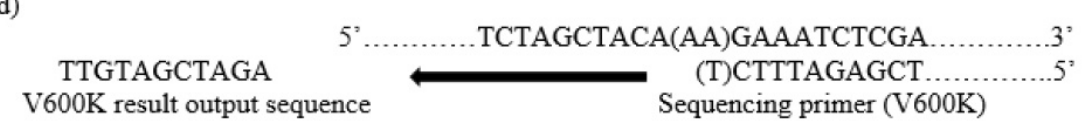

(e)

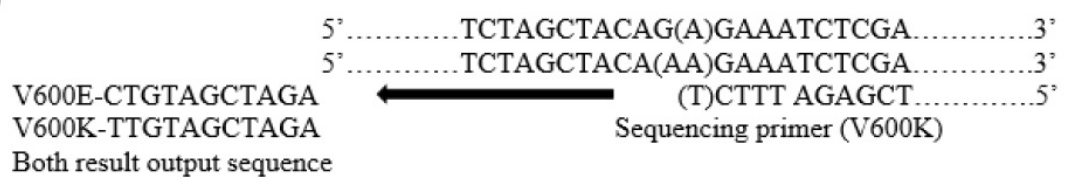

Figure 1. (a) Mutation locus of Braf p.V600E and Braf p.V600K. (b) Annealing of wild-type sequencing primer and generation of result output sequence CTGTAGCTAGA. (c) Annealing of Braf p.V600E mutant sequencing primer and generation of result output sequence CTGTAGCTAGA. (d) Annealing of Braf p.V600E mutant sequencing primer and generation of result output sequence TTGTAGCTAGA (Braf p.V600K). (e) Nucleotide result sequences from sample with both V600E/K. There will be two separate nucleotide sequences, one from V600E template and other from V600K template. The result output sequence will have two peaks at the first nucleotide and the rest are identical C/TTGTAGCTAGA. 
This manuscript presents the results of an investigative study with the following objectives: to determine 1) whether allele specific multiplex sequencing (ASMS) can detect Braf p.V600E mutations from a variety of solid tumors, 2) whether ASMS can detect all Braf p.V600E from samples that were positive for Braf p.V600E by SNaPshot or Ion Torrent, and 3) whether ASMS can detect Braf p.V600E among samples that were reported negative by SNaPshot or Ion Torrent. ${ }^{[15,16]}$

\section{MethodS}

\subsection{Samples}

DNA extracts from routine FFPE samples that were previously tested for Braf mutations were obtained from the Department of Pathology, Mount Sinai Hospital, New York, USA, and the Cross Cancer Institute, Edmonton, Alberta, Canada. None of the samples provided had patient information. Human Genomic Braf p.V600E reference standard DNA with 50\% Braf p.V600E (NM_004333.4:c.1799T > A, Braf: p.V600E) was obtained from Horizon Discoveries (Cambridge, UK), and wild-type human gDNA was also obtained (Promega). Human genomic DNA was extracted from wild-type human cell line NCI-PBCF-CCL247-HCT 116
(Horizon Discovery, UK. Cat No. HD-249) that is negative for Braf V600E/D/K/G and genomic DNA. Blood samples from four healthy subjects were also collected, and DNA was extracted using the DNeasy Blood and Tissue extraction kit (Qiagen).

\subsection{Sample preparation}

Forty-two FFPE DNA extract samples were received from the Department of Pathology, Mount Sinai Hospital, New York, USA. The samples were from melanomas, colorectal and lung carcinomas, brain tumors, and Langerhans cell histiocytosis. DNA was extracted from five sections of 5 micron FFPE from each sample using Maxwell extraction (Promega). Forty-one DNA samples were received from the Cross Cancer Institute, Edmonton, Alberta, Canada. Those samples were mainly from melanomas and colorectal carcinomas. Total DNA from each sample was extracted from five sections of 10 micron FFPE samples using QiaSymphony extraction (Qiagen). The tumor cell concentration of both sets of samples ranged from 10\%-90\%. DNA concentration of the extracted samples ranged from $1.4 \mathrm{ng} / \mu \mathrm{l}$ to $440 \mathrm{ng} / \mu \mathrm{l}$.

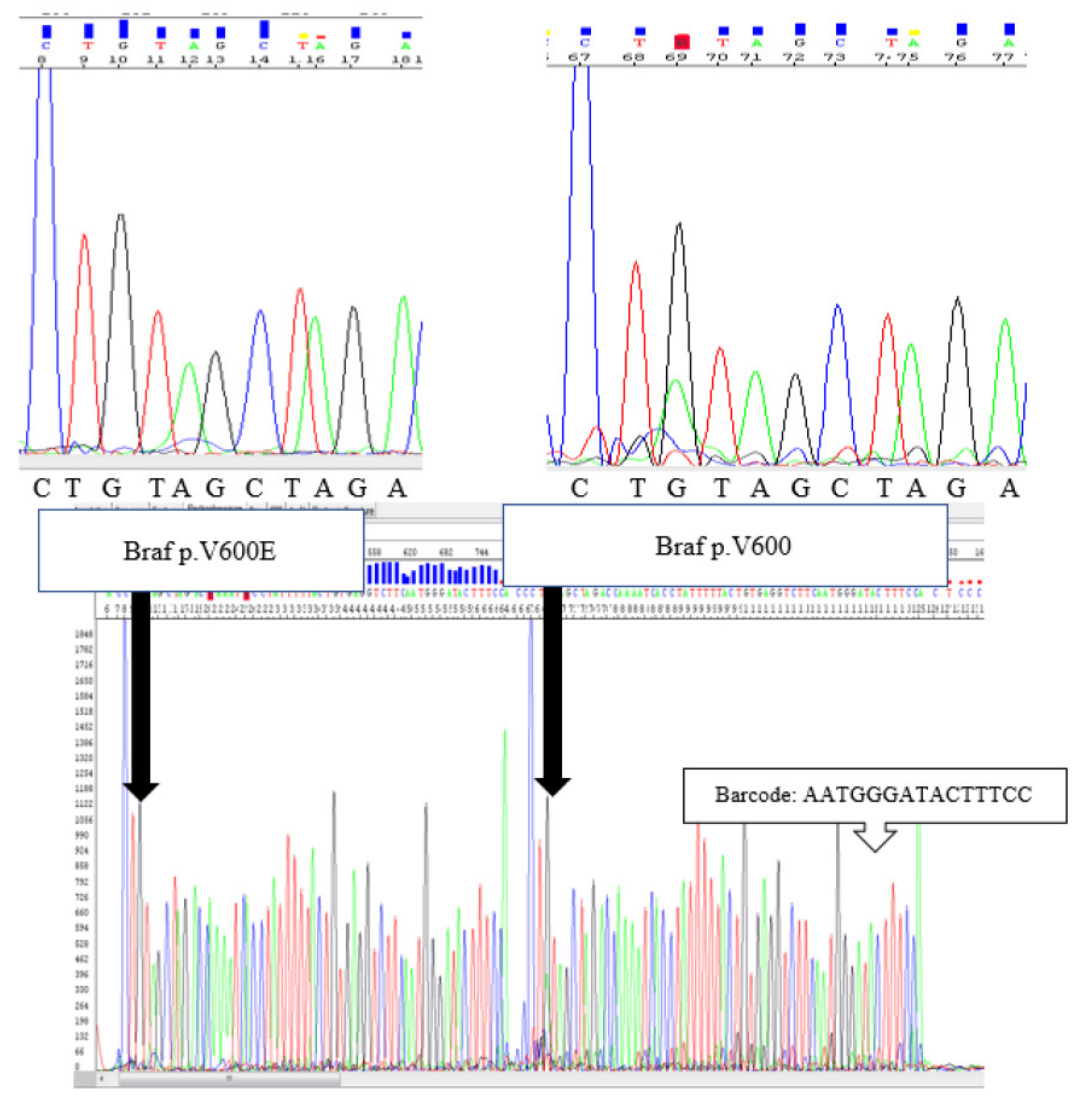

Figure 2. Electropherogram showing two separate and identical 11-nucleotide-long sequences CTGTAGCTAGA; one from Braf p.V600E (left) and one from the wild-type Braf p.V600 (right). Both these sequences also carry the same barcoding nucleotide sequence AATGGGATACTTTCC. 


\subsection{ASMS concept for Braf p.V600E}

Braf p.V600E is a single point mutation that changes glutamic acid for valine (1799 $\mathrm{T}>\mathrm{A}$; codon $\mathrm{GTG}>\mathrm{GAG}$ ) and Braf p.V600K is a single point mutation where (GTG > AAG) changes lysine for valine. The ASMS concept is based on amplifying a region encompassing the Braf p.V600E/K mutation site. In the absence of Braf p.V600E/K mutations, only the wild-type amplicon will be generated. If the sample has Braf p.V600E in the DNA extract, in addition to the wild-type Braf p.V600, a similar amplicon will be generated carrying the Braf p.V600E mutation. Hence, the Braf p.V600E sample will have two different amplicons, one from the mutant DNA and other from the wild-type. Both amplicons are of the same size and have the same nucleotide except one, the mutation (see Figure 1).
ASMS has two sequencing primers, one specific for the amplicon carrying the mutation that has ' $\mathrm{T}$ ' at its 3 ' end, and the other specific for the wild-type amplicon carrying ' $A$ ' at its 3' end. Further, each of these primers has a modification at the 5' end so that the cohort of truncated molecules generated from each sequencing primer do not overlap. ${ }^{[14]}$ The mutant sequencing primer (which is a part of the antisense strand) binds to the sense strand of the Braf p.V600E target, and generates a sequence (CTGTAGCTAGA) where ' $\mathrm{C}$ ' is the first nucleotide. Similarly, the wild-type sequencing primer (which is a part of the antisense strand) binds to the sense strand of the wild-type human DNA and generates a sequence (CTGTAGCTAGA) where ' $\mathrm{C}$ ' is also the first nucleotide (see Figure 2).
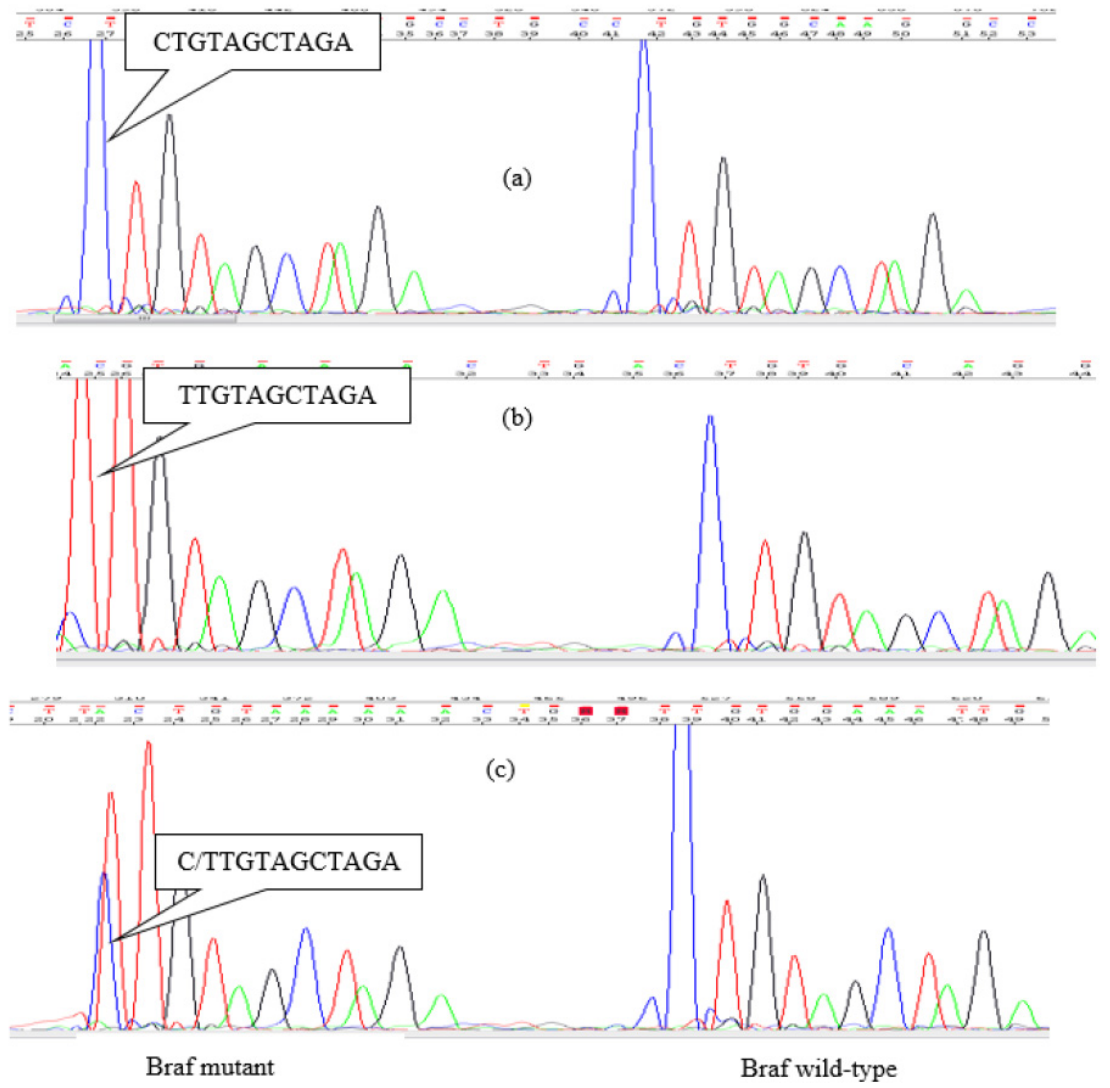

Figure 3. Electropherogram showing (a) Braf p.V600E, (b) Braf p.V600K and (c) Braf p.V600E and Braf p.V600K mutations.

In a sample that has Braf p.V600K, the very first nucleotide of the mutant read will be 'T' (TTGTAGCTAGA) (see Figure 3b).

If both Braf p.V600E and Braf p.V600K are present, there will be two clearly overlapping ' $\mathrm{C} / \mathrm{T}$ ' peaks at the first position (C/TTGTAGCTAGA), while the wild-type read will be (CTGTAGCTAGA ) (see Figure 3c). The nucleotide barcode is constructed by having a specific nucleotide sequence that denotes the source of the sample, test performed and the position in a 96 well plate. This is achieved by having a number of lower primers (e.g. 8), each having the same template specific annealing region, but with a specific nucleotide sequence at the 5' end that represents the source, test and position. (see Table 1). 
Table 1. Nucleotide bar code

\begin{tabular}{llll}
\hline Position & Customer ID & Test ID & Well ID \\
\hline Well 1 & AAT & GGGATAC & GGGCC \\
Well 2 & AAT & GGGATAC & AAACC \\
Well 3 & AAT & GGGATAC & TTTCC \\
Well 4 & AAT & GGGATAC & CCCCC \\
Well 5 & AAT & AAAATAC & GGGCC \\
Well 6 & AAT & AAAATAC & AAACC \\
Well 7 & AAT & AAAATAC & TTTCC \\
Well 8 & AAT & AAAATAC & CCCCC \\
\hline
\end{tabular}

Note. Test codes: Braf V600E=GGGATAC; Braf V600K=AAAATAC

ASMS is a platform technology that uses off-the-shelf equipment, commercially available reagents, and proprietary PCR primers and sequencing primers (MultiGEN Diagnostics, USA). The ASMS procedure includes total DNA extraction from FFPE samples, multiplex PCR, PCR clean up, cycle sequencing, dye clean up and injection into a Genetic Analyzer
(ThermoFisher, USA). ASMS is an open test system, where various steps to the procedure can be adjusted to increase sensitivity. This manuscript presents the standard procedure.

Multiplex Polymerase Chain reactions (MPCR) were set up for each sample. Each reaction mixture included $25 \mu \mathrm{l}$ of Master Mix 2X buffer (Qiagen), 10 pmoles each of the forward primer (5'GAAGACCTCACAGT AAA AATAGGTGATTTT GGT C-3') and reverse primer (5'TCAATGACTTTCTAGTTA ATCT CAGC AGCATCTC-3'), and $5 \mu \mathrm{l}-10 \mu \mathrm{l}$ of the DNA extract. MPCR conditions consisted of an initial denaturation at $95^{\circ} \mathrm{C}$ for $5 \mathrm{~min}$; 35 cycles of $95^{\circ} \mathrm{C}$ for 30 seconds, $60^{\circ} \mathrm{C}$ for 90 seconds, and $72^{\circ} \mathrm{C}$ for 30 seconds; and a final extension at $68^{\circ} \mathrm{C}$ for $10 \mathrm{~min}$. The amplicons were cleaned using Ampure (Beckman Coulter). Each batch had a negative control to ensure that there was no reagent contamination. Some of the PCR reactions included a specific reverse primer that carried a sample-tracking oligonucleotide sequence to track any possible cross-contamination.

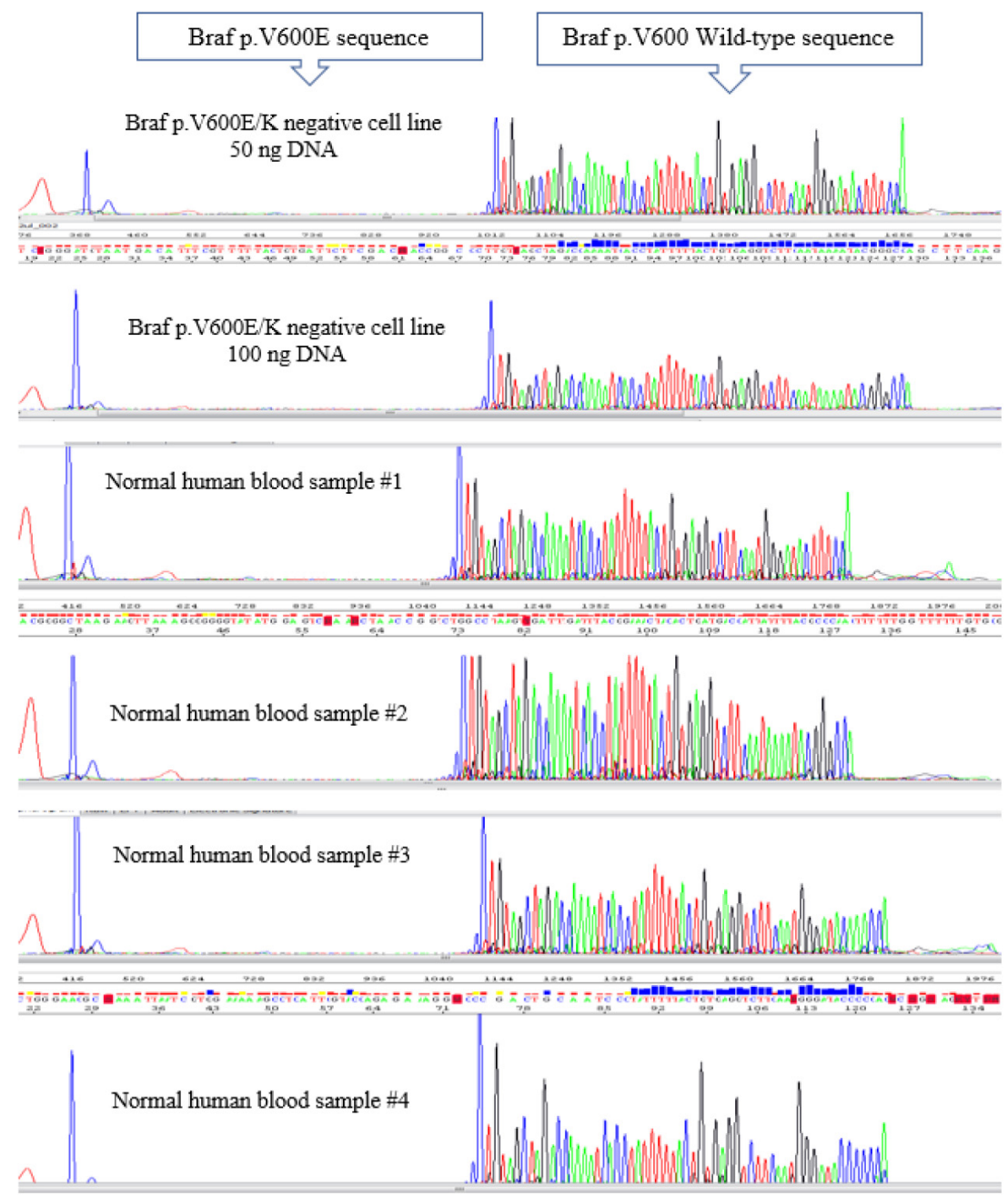

Figure 4. Top two electropherogram showing only Braf p.V600 wild-type from Braf p.V600E/K negative cell line. Last four electropherograms showing Braf p.V600 wild-type from normal human blood samples. 
Purified amplicons (35 $\mu \mathrm{l}$ ) were sequenced in a $50 \mu \mathrm{l}$ reaction volume using 1 pmol of Braf p.V600E sequencing primer (5'- $\square$-AACTGATGGGACCCACTCCATCG AGA TTTCT3') and Braf p.V600 sequencing primer (5'- $\square$-AAACTGAT GGGACCCACTCCATCGAGATTTCA-3') (MultiGEN Diagnostics), $3.5 \mu \mathrm{l}$ BigDye Terminator, $8.75 \mu \mathrm{l}$ of $5 \mathrm{X}$ Sequencing Buffer version 1.1 (Thermo Fisher Scientific), and $4 \mu \mathrm{l}$ water. The sequencing conditions consisted of 25 cycles of $96^{\circ} \mathrm{C}$ for $10 \mathrm{sec}, 52.5^{\circ} \mathrm{C}$ for $10 \mathrm{sec}$ and $60^{\circ} \mathrm{C}$ for $2.5 \mathrm{~min}$. Unincorporated dye terminators were removed using CleanSEQ (Beckman Coulter) and $10 \mu \mathrm{l}$ of the elute was added to $10 \mu \mathrm{l}$ of HiDi formamide. Samples were analyzed by capillary electrophoresis using the ABI PRISM Genetic Analyzer 3130 (Thermo Fisher Scientific). All electropherograms with a minimum of a fivenucleotide sequence (CTGTA) matching the target specified nucleotide sequence was recorded as positive.

\section{Results}

Human genomic DNA was extracted from human cell line NCI-PBCF-CCL247-HCT 116 (Horizon Discovery, UK. Cat No. HD-249) that is negative for Braf V600E/D/K/G, and two quantities were used (50 ng, $100 \mathrm{ng}$ ) in separate reactions. Both samples were negative for the Braf p.V600E/K mutation, and only positive for Braf V600 wild-type. In addition, genomic DNA from blood samples of four healthy subjects were tested using $50 \mathrm{ng}$ of total extracted DNA per reaction. All four samples were negative for the Braf p.V600E/K mutation, and only positive for the Braf V600 wild-type. This confirms that the method distinguishes between wild-type Braf and Braf p.V600E (see Figure 4).

The LLOD using 50\% Braf p.V600E gDNA from a cell line was $50 \mathrm{pg}$ of DNA (14 GE) (equivalent to 7.45 copies of Braf p.V600E and 7.45 copies of Braf p.V600) (see Figure 5).

(a)

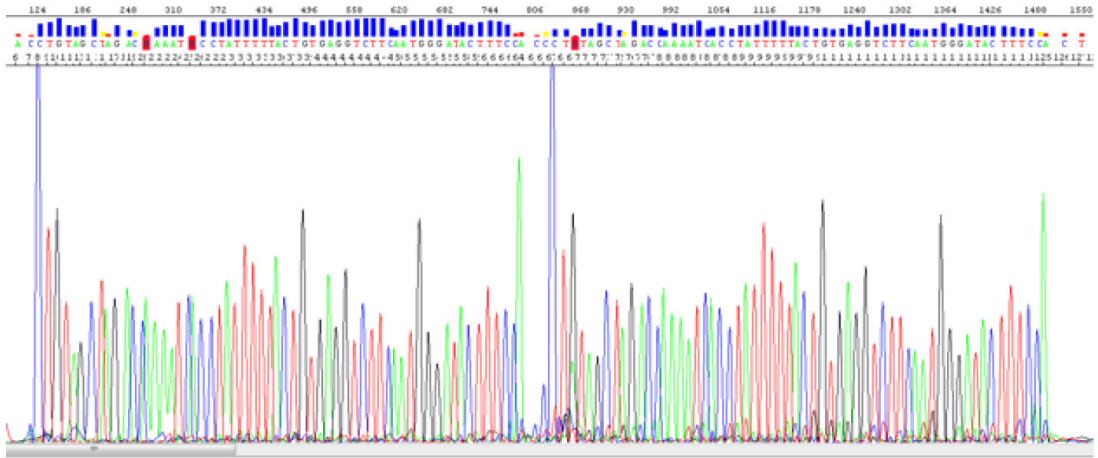

(b)

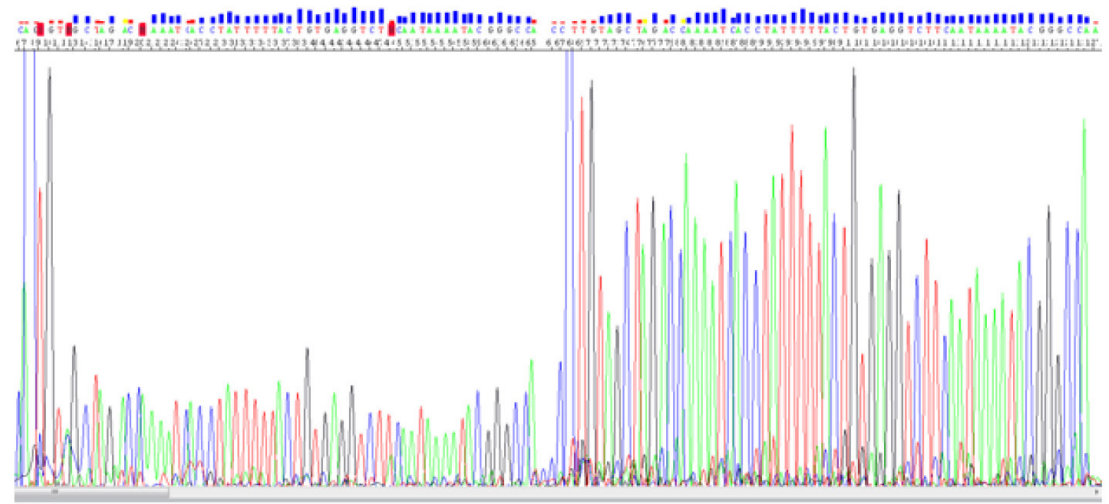

Figure 5. Electropherograms showing lower limit of detection from cell line carrying 50\% Braf p.V600E and 50\% Braf p.V600 (wild-type). (a) Samples with $0.05 \mathrm{ng}$ of total DNA (74 copies of Braf p.V600E and 74 copies of Braf p.V600), (b) Samples with $0.005 \mathrm{ng}$ of total DNA (7 copies of Braf p.V600E and 7 copies of Braf p.V600)

The ASMS assay was tested with DNA extracts from 42 samples that were previously tested by Ion Torrent. Out of 16 Braf p.V600E positive Ion Torrent samples tested by ASMS, 14 were positive for Braf p.V600E, one for Braf p.V600K alone by both ASMS and by Ion Torrent, and one was positive for both Braf p.V600E and Braf p.V600K by ASMS but positive for only Braf p.V600E by Ion Torrent. Out of the 26 negatives reported by Ion Torrent, 18 were positive for Braf p.V600E by ASMS and 8 were negative by ASMS (see Table 2). Out of the 21 samples that previously tested positive for Braf p.V600E by the SNaPshot assay, all 21 SNaPshot positives were positive for Braf p.V600E by ASMS. Out of the 
20 negatives reported by the SNaPshot assay, ASMS identi- can detect positives with only 40pg of the same DNA extract fied 10 samples as Braf p.V600E positive, and 10 samples (see Figure 6).

as Braf p.V600E negative. Dilution studies were performed on one of the positive samples, which showed that ASMS

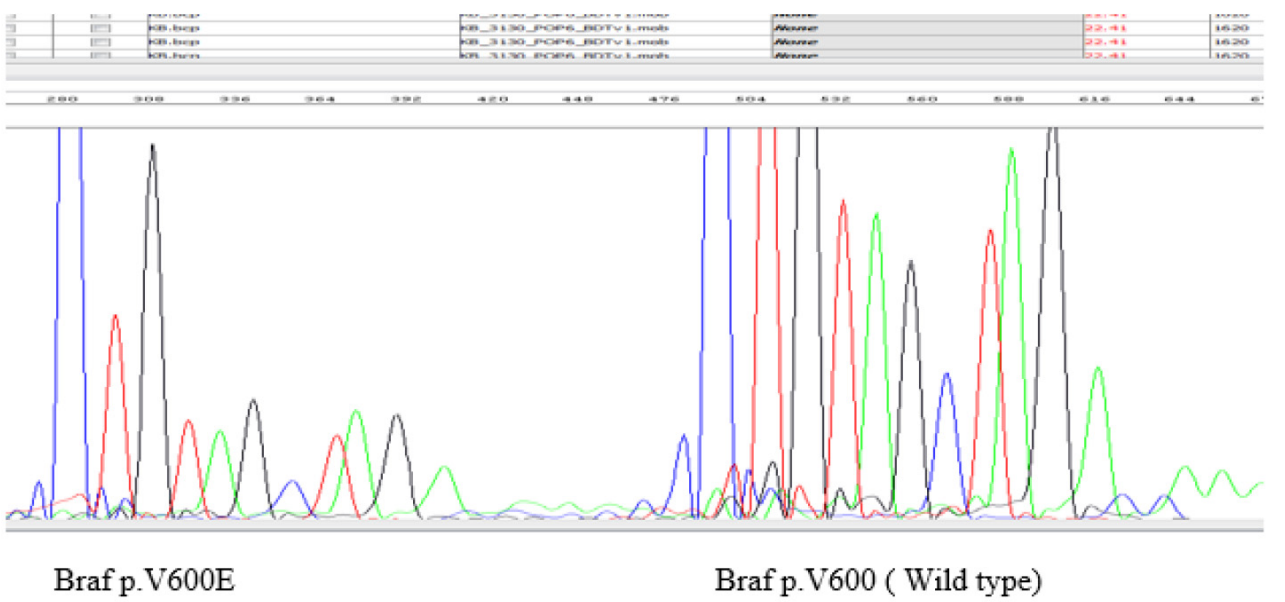

Figure 6. Electropherogram showing Braf p.V600E and Braf p.V600 at 1:1,000 dilution fo a Braf p.V600E positive clinical sample)

Table 2. Comparison of ASMS with Ion Torrent and SNapShot

\begin{tabular}{|c|c|c|c|c|}
\hline & \multirow{2}{*}{ No. Tested } & \multicolumn{2}{|l|}{ Positives } & \multirow{2}{*}{ Negatives } \\
\hline & & Braf p.V600E & Braf p.V600K & \\
\hline \multicolumn{5}{|l|}{ Ion Torrent } \\
\hline p.V600E positives & 15 & 15 & 1 & \\
\hline p.V600K positives & 1 & 0 & 1 & \\
\hline p.V600E/K negatives & 26 & 18 & 0 & 8 \\
\hline \multicolumn{5}{|l|}{ SNaPshot } \\
\hline p.V600E positives & 21 & 21 & & \\
\hline p.V600K positives & 0 & 0 & 0 & 0 \\
\hline p.V600E/K negatives & 20 & 10 & 0 & 10 \\
\hline
\end{tabular}

\section{Discussion}

ASMS showed $100 \%$ concordance in sensitivity with all samples that were Braf p.V600E positive tested by SNaPshot and Ion Torrent. However, ASMS results showed significant non-concordance in specificity with those from SNaPshot and Ion Torrent. To further confirm the specificity of the ASMS assay, we tested DNA extracted from a Braf p.V600E negative cell line and blood samples obtained from a normal population; all were negative for Braf p.V600E/K. These results, together with our earlier publication, ${ }^{[14]}$ confirms the specificity of ASMS assay. The ultra-specificity of the ASMS assay is attributed to the combination of the following features. First, the signal cohort generated by the mutant is separated from that of the wild type. This spatial separation on the same electropherogram eliminates any suppression of the mutant signal by the wild-type signal which occurs with conventional Sanger sequencing. The elimination of this suppression allows the mutant signal to be distinguishable from that of the wild type and baseline noise. Secondly, the simultaneous detection of the Braf p.V600 wild-type signal allows the wild type to act as an internal control. Thirdly, ASMS has a unique built-in oligonucleotide 'Barcode' that effectively monitors for the potential of cross-contamination during the testing process. These unique features, in combination, allow the ASMS Braf assay to reach higher specificity.

The result format of SNaPshot is a single molecular weightbased data point that cannot be verified as to authenticity of the target. Ion Torrent uses fragments of human genomic DNA as template, with nonspecific primers for amplification 
and sequencing, leading to several unavoidable variables in that process. To give allowance for uncertainty of the labeled probe used in SNaPshot, the number of variables, and the absence of target selection in Ion Torrent, both SNaPshot and Ion Torrent Braf p.V600E assays require a minimum of $40 \mathrm{ng}$ of total DNA extract per downstream processing. Forty nanograms of human DNA translates into more than 6,000 copies of Braf p.V600E in a sample for a positive assay. This approach is on par with the respective clinical claims for testing late stage (IV) melanoma, where the copy number is normally expected to be high. ASMS uses two Braf p.V600E specific PCR primers on a single piece of DNA template, and a Braf p.V600E specific sequencing primer, generating two Braf p.V600E sequences, one for Braf p.V600E amplicon (if positive) and one for Braf p.V600. Based on these assay characteristics, the ASMS process only requires a minimum of $40 \mathrm{pg}$ ( $\sim 6$ copies) of DNA extract to generate a positive Braf p.V600E signal.

The Braf p.V600E ASMS assay is a qualitative indication of the presence or absence of the mutation. Tumor mass is a dynamic heterogeneous cell population of clonal cancer cells, which are at various stages of transformation. Hence, the number of copies of the Braf p.V600E mutation could vary per defined sample volume throughout cancer progression, and be therefore unpredictable. Furthermore, there are two clinical scenarios where an ultra-sensitive test has potential benefit: 1) Biopsy samples, such as fine needle aspiration (FNA), in which the sample volume is limited, and 2) metastatic sites and nodal biopsies where the tumor mass is quite small.
Braf kinase is an intermediary protein in the complex cascade of the signal transduction pathway, mitogen-activated protein kinase (MAPK). The clinical significance of the Braf p.V600E/K mutations in metastatic melanoma, including use of inhibitors, are reported. ${ }^{[17-23]}$ Hence, low level detection of Braf p.V600E/K plays a pivotal role in the use of Braf kinase inhibitors. False negative Braf p.V600E/K results will prevent some patients from receiving treatment with kinase inhibitors, while at the same time allow for continuation of Braf p.V600E/V60K mediated intrinsic signal transduction in mutated cancer cells, potentially resulting in further proliferation of the tumor mass.

\section{Conclusion}

ASMS can detect Braf p.V600E from clinical samples. ASMS detected all positives that were detected by SNaPshot and Ion Torrent methods. Further, ASMS detected Braf p.V600E among those negatively reported by SNaPshot and Ion Torrent. Thus, ASMS has the potential to be a more sensitive platform to detect somatic point mutations in solid tumors.

\section{ACKNOWLEDGEMENTS}

We thank the Department of Pathology, Mount Sinai School of Medicine, 1425 Madison Ave, New York, NY 10029, and the Cross Cancer Institute, Edmonton, Alberta, Canada for providing DNA extracts from FFPE samples for this study.

\section{CONFLICTS OF INTEREST Disclosure}

Thuraiayah Vinayagamoorthy and Roger Hodkinson have financial interest in MultiGEN Diagnostics.

\section{REFERENCES}

[1] Cantwell-Dorris ER, O'Leary JJ, Sheils OM. BRAF V600E: Implications for carcinogenesis and molecular therapy. Mol. Cancer Ther. 2011; 10: 385-94. PMid:21388974. https://doi.org/10.1158/ 1535-7163. MCT-10-0799

[2] Ricci MS, Zong WX. Chemotherapeutic Approaches for Targeting Cell Death Pathways. The Oncologist 2006; 11: 342-57. PMid:16614230. https://doi.org/10.1634/theoncologist. 11-4-342

[3] Fan YS. Companion Diagnostic Testing for Targeted Cancer Therapies: An Overview. Genetic Testing and Molecular Biomarkers. 2013 July; 17(7): 515-23. PMid:23574530. https ://doi.org/10.108 9/gtmb. 2012.0510

[4] Kristian C, Lawrence MS, Carter SL, et al. Sensitive detection of somatic point mutations in impure and heterogeneous cancer samples. Nature Biotechnology. 2013; 31(3): 213-9. PMid:23396013. https://doi.org/10.1038/nbt.2514

[5] Zhang H, Song J, Ren H, et al. Detection of Low-Abundance KRAS Mutations in Colorectal Cancer Using Microfluidic Capillary Electrophoresis-Based Restriction Fragment Length Polymorphism Method with Optimized Assay Conditions. PLOS ONE. 2013; 8(1): e54510. PMid:23355875. https://doi .org/10.1371/journal. pone. 0054510

[6] Kevin Q, Qiulu P, Xi Z, et al. Detection of BRAF V600 Mutations in Metastatic Melanoma. Comparison of the Cobas 4800 and Sanger Sequencing Assays. The Journal of Molecular Diagnostics. 2013; 15(6): 790

[7] Bidshahri R, Attali D, Fakhfakh K, et al. Quantitative Detection and Resolution of BRAF V600 Status in Colorectal Cancer Using Droplet Digital PCR and a Novel Wild-Type Negative Assay. The Journal of Molecular Diagnostics. 2016; 18(2): 190-204. PMid:26762843. https://doi.org/10.1016/j.jmoldx.2015.09.003

[8] Schiefer A, Parlow L, Gabler L, et al. Multicenter Evaluation of a Novel Automated Rapid Detection System of BRAF Status in Formalin-Fixed, Paraffin-Embedded Tissues. The Journal of Molecular Diagnostics. 2016; 18(3): 370-7. PMid:26921540. https: //doi.org/10.1016/j.jmoldx.2015.12.005

[9] Sakai1 K, Tsurutani J, Yamanaka T, et al. Extended RAS and BRAF Mutation Analysis Using Next-Generation Sequencing. PLOS ONE 2014; 10(5): e0121891.

[10] Huang T, Zhuge J, Zhang WW. Sensitive detection of BRAF V600E mutation by Amplification Refractory Mutation System (ARMS)- 
PCR. Biomarker Research. 2013; 1: 3. PMid:24252159. https : //doi.org/10.1186/2050-7771-1-3

[11] Magnin S, Viel E, Baraquin A, et al. A Multiplex SNaPshot Assay as a Rapid Method for Detecting KRAS and BRAF Mutations in Advanced Colorectal Cancers. The Journal of Molecular Diagnostics. 2011; 13(5): 485-92. PMid:21742054. https ://doi .org/10.101 $6 / j \cdot j \operatorname{moldx} .2011 .05 .010$

[12] Gong RX, Gong YP, Yang J, et al. Efficient detection of the V600E mutation of the BRAF gene in papillary thyroid carcinoma using multiplex allele-specific polymerase chain reaction combined with denaturing high-performance liquid chromatography. Genetics and Molecular Research. 2013; 12(4): 4990-7. PMid:24301760. https://doi.org/10.4238/2013.0ctober.24.11

[13] Vargas DY, Kramer FR, Tyagi S, et al. Multiplex Real-Time PCR Assays that Measure the Abundance of Extremely Rare Mutations Associated with Cancer. PLOS ONE. 2016; 11(5): e0156546. PMid:27244445. https://doi.org/10.1371/journal. pone.0 156546

[14] Yo J, Hay KSL, Vinayagamoorthy D, et al. Detection of BRAF mutations from solid tumors using TumorPlexTM technology. Methods X. 2014; 15(C): 316-22.

[15] Magnin S, Viel E, Baraquin A, et al. A Multiplex SNaPshot Assay as a Rapid Method for Detecting KRAS and BRAF Mutations in Advanced Colorectal Cancers. J Mol. Diagn. 2011; 1: 485-92. PMid:21742054. https://doi.org/10.1016/j.jmoldx. 2011. 05.010

[16] Romano R, Pradervand S, Paillusson A, et al. Identification of Multiple Mechanisms of Resistance to Vemurafenib in a Patient with BRAF V600E-Mutated Cutaneous Melanoma Successfully re-challenged after Progression. Clin Cancer Res. 2013; 19(20): 5749-57. PMid:23948972. https : //doi .org/10.1158/1078-0 432. CCR-13-0661

[17] Ascierto PA, Kirkwood JM, Grob JJ, et al. The role of BRAF V600 mutation in melanoma. J Transl Med. 2012; 10: 85. PMid:22554099. https://doi.org/10.1186/1479-5876-10-85

[18] Chen RE, Thorner J. Function and Regulation in MAPK Signaling Pathways. Biochim Biophys. Acta. 2007; 1773(8): 1311-40. PMid:17604854. https ://doi.org/10.1016/j.bbamcr. 2007. 05.003

[19] Davies H, Bignell GR, Cox C, et al. Mutations of the BRAF gene in human cancer. Nature. 2002; 417: 949-53. PMid:12068308. https : //doi.org/10.1038/nature00766

[20] Dong J, Robert GP, Qiao R, et al. BRAF Oncogenic Mutations Correlate with Progression rather than Initiation of Human Melanoma, Cancer Research. 2003; 63: 3883-5. PMid:12873977.

[21] Kumar R, Angelini S, Czene K, et al. BRAF Mutations in Metastatic Melanoma: A Possible Association with Clinical Outcome. Clinical Cancer Research. 2003; (9): 3362-8. PMid:12960123.

[22] Chapman PB, Hauschild A, Robert C, et al. Improved Survival with Vemurafenib in Melanoma with BRAF V600E Mutation. N Engl J Med. 2011; 364: 2507-16. PMid:21639808. https ://doi .org/10 .1056/NEJMoa1103782

[23] Caroline R, Karaszewska B, Schachter J, et al. Improved Overall Survival in Melanoma with Combined Dabrafenib and Trametinib. N Engl. J Med. 2015; 372: 30-9. PMid:25399551. https: //doi.org/10.1056/NEJMoa1412690 